\title{
Identifying Suitable Projection Parameters and Display Configurations for Mobile True-3D Displays
}

\author{
Marcos Serrano $^{1,2}$, Dale Hildebrandt ${ }^{1}$, Sriram Subramanian ${ }^{3}$, Pourang Irani ${ }^{1}$ \\ ${ }^{1}$ University of Manitoba \\ ${ }^{2}$ IRIT - University of Toulouse \\ ${ }^{3}$ University of Bristol \\ Winnipeg, MB, R3T 2N2, Canada \\ Toulouse, 31062, France \\ marcos.serrano@irit.fr \\ Woodland Road, BS81UB UK \\ sriram@cs.bris.ac.uk
}

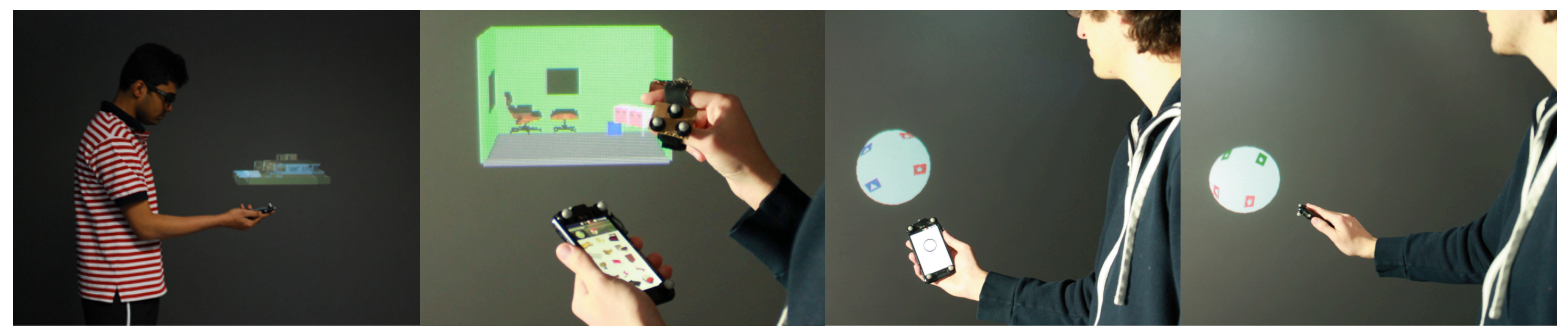

Figure 1. a) Illustration of a true-3D visualization on a mobile device. b) We implemented a 3D interior design application in our true-3D mobile prototype. c-d) Subject doing visual search during the first experiment.

\begin{abstract}
We present a two-part exploration on mobile true-3D displays, i.e. displaying volumetric $3 \mathrm{D}$ content in mid-air. We first identify and study the parameters of a mobile true3D projection, in terms of the projection's distance to the phone, angle to the phone, display volume and position within the display. We identify suitable parameters and constraints, which we propose as requirements for developing mobile true-3D systems. We build on the first outcomes to explore methods for coordinating the display configurations of the mobile true-3D setup. We explore the resulting design space through two applications: 3D map navigation and 3D interior design. We discuss the implications of our results for the future design of mobile true-3D displays.
\end{abstract}

\section{Author Keywords}

Mobile True-3D, intangible displays, mid-air pointing, displays.

\section{ACM Classification Keywords}

H.5.2. Information interfaces and presentation: Interaction.

\section{INTRODUCTION}

Benefiting from 3D on mobile devices is pertinent, beyond video games, for mobile scenarios such as 3D interior design or 3D map exploration. Mobile devices (smartphones and gaming consoles) incorporate at present

Permission to make digital or hard copies of all or part of this work for personal or classroom use is granted without fee provided that copies are not made or distributed for profit or commercial advantage and that copies bear this notice and the full citation on the first page. Copyrights for components of this work owned by others than ACM must be honored Abstracting with credit is permitted. To copy otherwise, or republish, to post on servers or to redistribute to lists, requires prior specific permission and/or a fee.

MobileHCI '14, September 23 - 26 2014, Toronto, ON, Canada

Copyright 2014 ACM 978-1-4503-3004-6/14/09 \$15.00.

http://dx.doi.org/10.1145/2628363.2628375 auto-stereoscopic displays as a first step toward achieving true-3D content [21]. True-3D, i.e. displaying volumetric 3D content in mid-air, can be accomplished through different means: stereoscopic displays with head-tracking, optical illusions, moving parts or augmented reality glasses $[14,15,17,20,27]$. Integrating true-3D on mobile devices, apart from facing hardware challenges, presents a number of unresolved human factors questions concerning its use.

We possess limited knowledge on both the numerous constraints imposed on viewing and interacting with mobile true-3D interfaces and the usage scenarios suitable for such displays. These include knowing about the ideal angular position, size and distance of the volumetric projection, relative to the mobile device, the projection limits on visual search and direct interaction and how to coordinate the mobile and true-3D views. Answers to such questions will equip manufacturers and designers with tools to begin exploring a range of technologies that can be tailored for true-3D input on mobile devices.

We study the various factors that can potentially influence the effective deployment of true-3D on mobile devices in an emulated environment (Figure 1). We focus on mobilemounted 3D projection, which means that the true-3D projection moves with the mobile device as if both were attached. We systematically address the above listed fundamental questions for this form of Mobile MultiDisplay Environment (MMDE).

Our contributions include: 1) an identification of suitable display parameters and constraints for true-3D mobile display; 2) an exploration of the coordinated display configurations for the 2D mobile and true-3D displays; and 3 ) the application of these configurations to the design of two proof-of-concept applications, a 3D map and a 3D interior design application (Figure 1). 


\section{STATE OF THE ART}

\section{True-3D}

True-3D refers to any 3D digital display capable of producing mid-air, full-depth-cue (or volumetric), multiangle and/or multi-user images without the need for user instrumentation $[15,17]$.

Recent work studying the value of 'true' 3D displays has relied on emulation for recreating this type of display $[1,15,20]$. To produce intangible and mid-air images, most systems use optical illusions. Vermeer is an interactive $360^{\circ}$ viewable $3 \mathrm{D}$ display based on the optical illusion of using two parabolic mirrors [2]. HoloDesk is a system that exploits a see through display with kinetic motion for creating a 'true' 3D effect [15]. Virtual Reality (VR) has mostly emulated the 'true' $3 \mathrm{D}$ experience using stereoscopic projections, an approach we adopt to evaluate the display projection parameters for true-3D mobile displays. Head-mounted augmented reality systems have largely evolved and can facilitate 3D stereo viewing capabilities, with lighter glasses and see-through displays, such as the Vuzix Star 1200 [28]. Depth cues can be produced using auto-stereoscopic displays, which have appeared on mobile and handheld gaming devices. Such screens can be viewed by only one user and have a narrow viewing angle. Coupling such displays with head-tracking allows various angles of view, as in Fuwa-Vision [20], an auto-stereoscopic display presenting images in mid-air.

Aside from good 'emulations', the last few years witnessed 'true' 3D displays developing at an accelerated pace. The HelioDisplay generates a mid-air display using floating particles as a projection surface [14], but is limited to projection on a two-dimensional plane. Researchers more recently have demonstrated the use of laser plasma technology to produce 3D-objects with light dot arrays in space [17]. This method does not allow for direct interaction with the true-3D display.

While such advances bring us closer to experiencing true$3 \mathrm{D}$, we possess limited knowledge on how to design for such displays to co-exist with other forms of displays, such as mobile devices. To our knowledge, this work is the first exploration of the human factors considerations for a true3D mobile multi-display environment.

\section{Mobile Multi-Display Environments}

Previous works have classified Mobile Multi-Display Environments (MMDE) on a continuum from being partially mobile (one mobile phone used with fixed displays [1]) to fully mobile systems [5]. Fully mobile can be further divided into multi-device-single-display and single-devicemulti-display [5] MMDEs. We focus our explorations on the category of fully mobile, single-device and multidisplay systems.

Foldable mobile devices such as the Nintendo 3DS [21] integrate a second screen to extend its display capabilities. Using an additional screen has the disadvantage of increasing the overall size and weight of the device. Picoprojectors alleviate the above problem by allowing use of a secondary display as needed [25]. Their main limitations are the need for a flat projection surface and the large distance between the projected display and the main display. While the latter drawback can be resolved by finding a closer projection surface [5] or by using steerable projectors [6], the former still limits where such systems can be used. The use of a true-3D display could overcome this limitation.

\section{Interaction with mobile 3D and true-3D interfaces}

Recent work demonstrates methods for interacting with 3D content using different smartphone interaction capabilities such as the touchscreen, the accelerometer or the camera $[29,18]$. Song et al. presented a set of techniques for visual exploration of volumetric data [29]. PalmSpace proposes the use of continuous around-device gestures for $3 \mathrm{D}$ rotation tasks [18]. Sitcky tools are a set of multi-touch techniques for 6DOF manipulation on flat tabletops [13]. However it is unobvious whether such solutions transfer well to handheld mobile true-3D, where the $3 \mathrm{D}$ content moves with the device.

In the case of true-3D displays, we can borrow knowledge from the literature on 3D user interfaces [11,19]. However, with actual systems very few results exist. Chan et al. investigated direct-touch interaction for 2D intangible displays [7]. The apparatus was based on the use of a Fresnel lens to create the optical illusion of true-3D. Results showed users performed poorly in distinguishing the $\mathrm{z}$ coordinate of the targets. The use of visual pseudo-shadow feedback improved user performance. Vermeer [1] and HoloDesk [15] present interactions with holographic images, such as direct-touch, scooping and grasping. The growing body of literature on 3D user interfaces [12, 13] inspires the design of the basic direct input methods we developed in our prototype applications.

\section{DISPLAY PROPERTIES}

The following factors, which we derive in part from the MMDE literature, can influence the display properties of a true-3D mobile multi-display environment.

\section{Projection area}

While pico-projectors need a projection surface, true-3D projectors may display an image in mid-air around the mobile device. Prior work has generally kept the mobile projection pointing downward [5], straight [30] or steerable (using a motor to direct the projector) [6]. These solutions provide significant flexibility in finding a suitable projection surface. A true-3D mobile display needs not be constrained by the position of the projection throw. Therefore after considering the potential projection areas around the smartphone, we decided to focus on the top area of the phone (Figure 2-left). This area always remains visible when the user rotates the phone to inspect the $360^{\circ}$ true-3D image. 


\section{Projection distance to the 3D object}

The distance between the mid-air 3D projection area and the smartphone (Figure 2-center) may have an impact on users' visual perception and direct input. If the projection is far from the device, it may affect the continuity of the visual search [5] but even further limit direct-touch interaction with the true-3D and require indirect forms of interaction. Previous research has investigated the effect of visual separation (angle and distance between displays) on MMDE [5]. Results show there was no effect on task performance but a higher number of eye context switches occurred if both displays were not in the same field of view (approximately 30\% higher). We further investigate this factor in our first experiment.

\section{Projection angle}

We define the projection angle as the angle between the phone's $y$-axis and the $3 \mathrm{D}$ object. Traditional depictions of mobile true-3D envision the $3 \mathrm{D}$ content at a $90^{\circ}$ angle relative to the phone's plane (Figure 2-right) or displayed directly over the touchscreen (see Samsung's concept vision in [26]). These depictions assume the best projection 'extends' the mobile display into a 3D volume. However, this projection style presents several drawbacks. First, it is unclear how both displays would properly overlay as there could be issues with color mixing and light intensity since the touchscreen would likely be brighter than the true-3D. Second, this vision is limited as it considers the true-3D simply as an extension of the touchscreen instead of viewing it as a secondary display that can extend the mobile phone's capabilities. Our first experiment examines this factor, studying angles from $90^{\circ}$, perpendicular to the phone's plane, to $0^{\circ}$, collinear to the phone's plane (Figure 2-right).

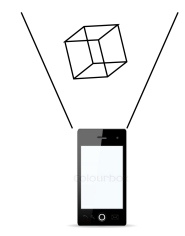

projection area

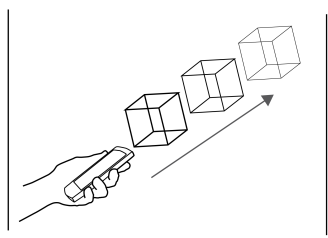

projection distance

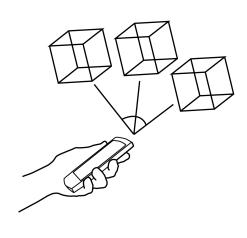

projection angle
Figure 2. Projection area, distance and angle properties.

\section{Projection volume}

On traditional MMDE, display sizes are heterogeneous (i.e. pico-projection and mobile touchscreen will vary in size) and further can change while being used if the device is hand held. Some studies with fixed displays show better performance for identical size compared to different size displays [5,24]. In the case of mobile true-3D, its volume may affect visual search as well as direct-touch interaction, a factor we investigate in our experiment.

\section{Point-of-view}

The visual exploration of a mobile true-3D display can rely on wrist rotation dexterity to avoid complex interactions for rotating it. There are three main rotation axes around the wrist: flexion/extension, pronation/supination and ulnar/radial deviation. Previous work on wrist dexterity has identified that the maximum angles are $60^{\circ}-45^{\circ}$ for flexion/ extension, $65^{\circ}-60^{\circ}$ for pronation/supination, and $15^{\circ}-30^{\circ}$ for ulnar/radial deviation [23]. We thus expect limited accessibility to occluded areas on the true-3D projection, which we explore across the entire $360^{\circ}$ true-3D display using wrist dexterity alone.

\section{MOBILE MULTI-DISPLAY TRUE-3D PROTOTYPE}

To gain an understanding of the various properties that could influence the human factors aspects with mobile multi-display true-3D we implemented a running emulation.

\section{Prototype}

Our implementation was based on a stereoscopic display coupled with head tracking, on the VisCube [27] platform, an immersive environment composed of a projection wall and floor (Figure 3). In this system the user has to wear polarized glasses with IR markers to allow visual headtracking. 3D content was developed using GLUT. We used an LG-P925 smartphone (4.3', screen, weight 6oz, dimensions $5 "(\mathrm{H}) \times 2.7 "(\mathrm{~W}) \times 0.4 "(\mathrm{D}))$ running Android 2.3. The position of the mobile device in the environment was tracked using a Vicon IR motion tracking system and IR markers.

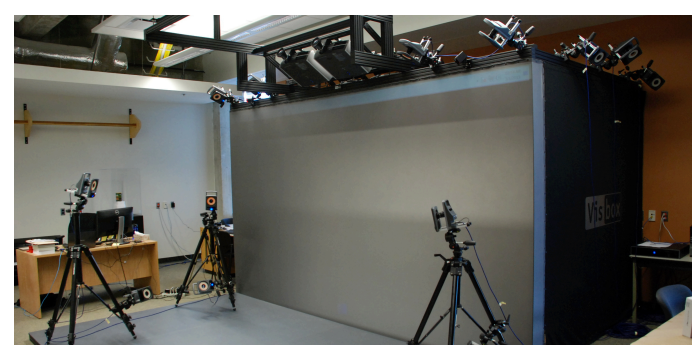

Figure 3. We built our prototype in a VisCube 3D [27] immersive system, tracking the mobile device using optical tracking.

\section{Emulating True-3D}

Emulating true-3D in a CAVE environment has been achieved by others. The Personal Cockpit project investigated the use of virtual windows around the user through emulation in a VisCube immersive system [9].

\section{EXPERIMENT 1: DISPLAY PROPERTIES}

The goal of this experiment was to identify the best spatial configuration for the projection of a mobile true-3D display to ensure effective visual search. We explore the case of natural user interaction, i.e. wrist rotation for search without interface support, but do not explore any type of user input. We focus on the properties described in the previous section (Figure 2): projection's angle to the phone plane, distance to the phone, volume and pattern position on the true-3D (point-of-view).

\section{Apparatus, task and instructions}

We used the VisCube prototype described previously. Participants were required to identify the location of a 
graphical pattern on a 3D opaque sphere on the true-3D display. The sphere was separated into eight parts, each one containing a unique pattern. All eight patterns had the same volume and were symmetrically positioned on the sphere (Figure 4).

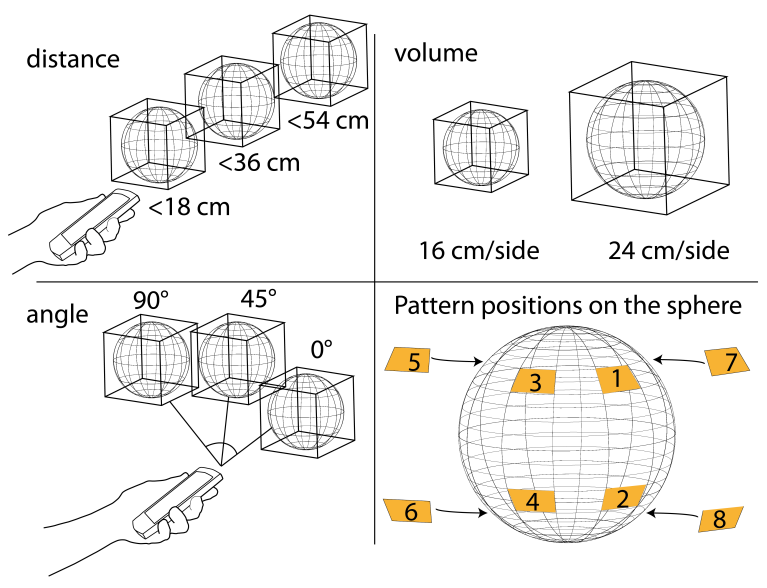

Figure 4. Properties evaluated in the first experiment: true-3D display's distance to the phone, volume size, angle to the phone and item position on the sphere (4 positions on the front and 4 symmetrical positions on the back).

We used a basic geometrical shape as the stimulus pattern: circle, triangle, square or star. The stimulus was displayed on the mobile screen to ensure that all users would start the task with a focus at the same location. Once the stimulus was displayed, in black and white, users had to find its coloured version on the sphere (three possible colors) and click a button of the same color on a wand (held with the other hand) to select the answer (Figure 1). Color allowed differentiating correct and false answers with minimum cognitive load. We used the wand merely as an experimental input to collect answers from users. Buttons on the wand are disposed overall at the same distance of the thumb. In case of an error, we recorded the number of attempts it took the user to find the correct answer. We asked participants to minimize selection time (primary demand) without neglecting the error rate (secondary demand).

The position of the sphere was determined by the angle and distance factors (Figure 4). The angle represents the position of the sphere in relation to the mobile device plane: $0^{\circ}, 45^{\circ}$ and $90^{\circ}$. We defined three approximate distances to the center of the sphere: close when the sphere center is $18 \mathrm{~cm}$ away from the top of the device, middle when it is $36 \mathrm{~cm}$ away and far when it is $54 \mathrm{~cm}$ away. The volume factor represents the volume of the sphere's bounding cube (Figure 4): the small cube was approximately $16 \mathrm{~cm} / \mathrm{side}$ and the big cube $24 \mathrm{~cm} /$ side.

\section{Participants}

11 men and 1 woman, aged 25.25 years on average, volunteered for the experiment. All of them were right handed; 9 held the mobile device in the right hand while 3 preferred to hold it with the left hand. We mirrored the results from those 3 participants for the position factor (others factors not being dependent on the hand used).

\section{Procedure}

We used a $3 \times 3 \times 2 \times 8$ within-subject design with Angle $\left(0^{\circ}\right.$, $45^{\circ}$ and $90^{\circ}$ ), Distance (close $<18 \mathrm{~cm}$, medium $<36 \mathrm{~cm}$ and far $<54 \mathrm{~cm}$ ), Volume ( small $=16 \mathrm{~cm} /$ side and $\mathrm{big}=24 \mathrm{~cm} / \mathrm{side}$ ) and Item Position ( 8 areas of the sphere) as factors. We ran three blocks of trials for each condition. Angle was counterbalanced using a $3 \times 3$ Latin square while other factors were presented randomly. We ran one training block before the experiment. The experiment consisted of 144 conditions $\times 3$ blocks $\times 12$ users $=5184$ trials.

\section{Data Collection}

We recorded the smartphone's position and angle with the Vicon. Besides success rate, we measured trial completion time, from stimulus onset to button pressed. Participants filled a 5-point Likert scale with nine questions to indicate preference for the four factors. The ninth question asked whether users liked the concept of mobile true-3D.

\section{Results and Discussion}

We used the univariate ANOVA with post-hoc comparisons using the Bonferroni adjustment for all our analyses. Results reveal a main effect of angle $\left(F_{2,22}=97.6, p<.001\right)$, distance $\left(F_{2,22}=91.6, p<.001\right)$ and position $\left(F_{7,77}=139.7\right.$, $p<.001)$ on completion time. There was no consistent effect of volume $\left(F_{1,11}=.116 p=.734\right)$. We found an interaction between angle and distance $\left(F_{4,44}=12.6, p<.001\right)$ and volume and distance $\left(F_{2,22}=5.5, p=.004\right)$. We also found interaction effects for position and angle $\left(F_{14,154}=11.7\right.$, $p<.001)$ and position and distance $\left(F_{14,154}=11.6, p<.001\right)$. We did not find any main effects for accuracy rate, with an average success rate of $97.8 \%$ (std. dev. $0.2 \%$ ).
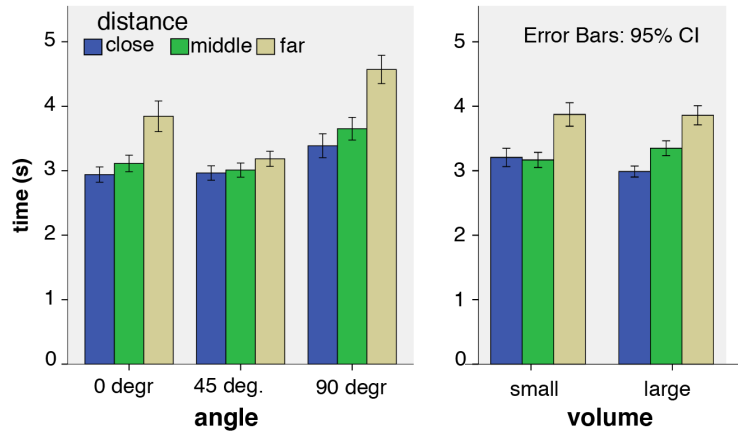

Figure 5. Completion time (s) for Distance at each Angle (left) and Distance at each Volume (right).

Angle and distance interaction: Overall, completion time increased with distance and angle (Figure 5). Post-hoc analysis revealed a significant difference between all three angle positions, $45^{\circ}$ (3.0s on average) being slightly faster than $0^{\circ}(3.3 \mathrm{~s})$ and considerably faster than $90^{\circ}(3.8 \mathrm{~s})$. Surprisingly, the worst projection angle for a visual search task on a mobile true-3D display is $90^{\circ}$, which contradicts popular conceptions of such displays. Subject 4 reported 
"when the sphere is farther horizontally it is easier to search than if it is vertically distant". Concerning distance, we also found a significant effect between all three distances. Performance with the close (3.0s) being slightly better than with middle (3.2s) and considerably better than far (3.8s). The results do not reveal a linear degradation of distance on visual search (while distances values are linear) and can thus expect a stronger negative impact with further positions. Concerning the interaction between both factors, we notice in the case of $45^{\circ}$ that the difference between all three distances is less important than for the other two angles, which are particularly bad when the $3 \mathrm{D}$ image is far.

Position on the sphere: As expected, the front hemisphere is significantly faster than the back (2.7s vs. $4 \mathrm{~s})$. Our experiment required searching for objects in the back part of the projection to capture conditions such as 3D object occlusion. We find a significant difference between the back right (3.7s) and left elements (4.4s) (Figure 6). Several users reported severe difficulty reaching the back left elements and particularly the top left object (4.6s) due to a physiological limitation in wrist rotation. This result is consistent with previous works on wrist rotation [23].

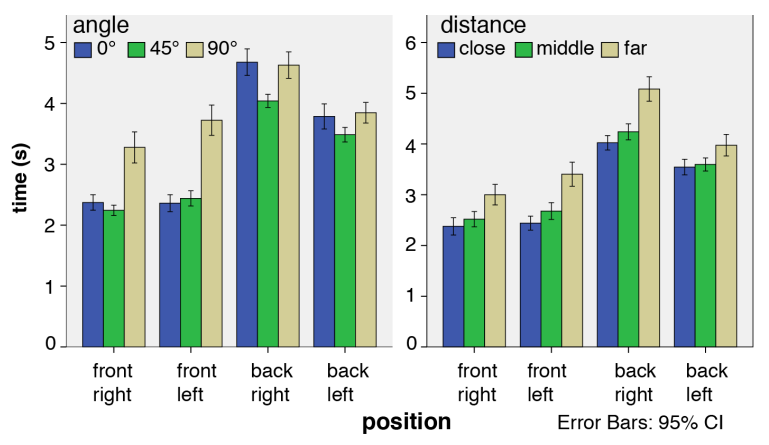

Figure 6. Completion time (s) for Position at each Angle (left) and Position at each Distance (right).

\section{User Preference}

From the 5-point Likert scale most participants found it difficult to search for content on the large sphere (Q1), did not show a specific preference for projection at $0^{\circ}$ or $45^{\circ}$ (Q7), and generally preferred the closer distance (Q6). All participants liked the concept of having mobile true-3D.

\section{Summary of Experiment 1 Results}

This first study demonstrates that a $3 \mathrm{D}$ object positioned at an angle of $0^{\circ}$ or $45^{\circ}$ and at a distance of less than $36 \mathrm{~cm}$ (middle or closer) performs best for visual search. We also found that the region in the back and opposite to the hand holding the device is the weakest for object search. This is primarily due to the wrist dexterity as observed during the experiment and from participant feedback. Wrist dexterity also affects objects located further away, i.e. these become hard to inspect under all angles. Thus, our results recommend shorter distances if the device is to solely rely on wrist rotation for viewing the display. We consider these constraints in experiment 2 .

\section{EXPERIMENT 2: DIRECT-TOUCH INTERACTION}

The goal of this experiment is to investigate the effect of volume size on visual search and direct input.

\section{Task and instructions}

Participants were required to identify the location of a graphical pattern on the true-3D display and to select it with direct mid-air touch. To complete the task, they used their finger, equipped with IR markers, to touch the pattern on the sphere. We added one larger volume size than in experiment 1 to further investigate this factor (note we did not see effects of the volume sizes selected in the previous study). The small volume was approximately $16 \mathrm{~cm} /$ side, the medium $24 \mathrm{~cm} /$ side and the large $36 \mathrm{~cm} /$ side (the volume is constant and does not scale to the distance to the user).

\section{Participants}

Ten men and two women, of an average age of 26.3 years volunteered for the experiment. Five participated in the first experiment. Eleven were right handed and one left-handed. They all held the mobile device with their left hand and used their right index finger for interaction.

\section{Procedure and data collection}

This experiment followed a $3 \times 2 \times 8$ within-subject design with Volume (small, medium and large), Angle $\left(0^{\circ}\right.$ and $45^{\circ}$ ) and Item Position ( 8 areas of the sphere) as factors. We set the Distance factor to middle $(<36 \mathrm{~cm})$ to allow enough space for the large Volume to be displayed. Three blocks of trials were run for each condition, the angle factor being counter-balanced while others were selected randomly. We also ran one training block before the experiment to reduce the learning effect between new participants and those who participated in the first study. The experiment consisted of 48 conditions $\times 3$ blocks $\times 12$ users $=1728$ trials. We

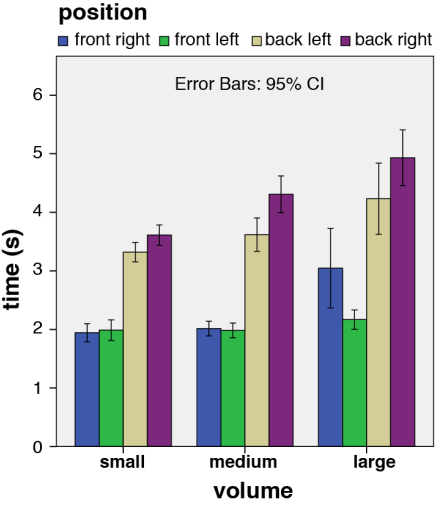

Figure 7. Completion time (s) for Volume at each Position. collected the same data as in the previous experiment.

Results and Discussion We used the univariate ANOVA with post-hoc comparisons using the Bonferroni adjustment for all our analyses. Results revealed a main effect of volume $\quad\left(F_{2,22}=8.4\right.$, $p<.001)$ and of position $\left(F_{7,77}=38.8, \quad p<.001\right) \quad$ on completion time. We found an interaction between volume and position $\left(F_{14,154}=2.5, p=.002\right)$. There was no consistent effect of angle. Concerning error rate, there was a significant effect of volume $\left(F_{2,22}=9.6, p<.001\right)$ but no significant effect of other factors (position and angle).

Volume: Overall, completion time increased with the volume of the display (Figure 7). A post-hoc analysis revealed there is no significant difference between the mean 
time for the small $(2715 \mathrm{~ms})$ and the medium volumes (2980 ms). There was however a significant difference between those two times and the mean time for the large volume (3594 ms). Results showed that success rate increased with volume: $91.3 \%$ for the small volume, $95.8 \%$ for the medium volume and $97.2 \%$ for the larger volume. Most of the errors were due to the intangible nature of the display. In some cases users had a bad perception of 3D depth and touched a back or adjacent item while pointing: this happened more often on the small volume, since items were closer, thus explaining the difference in success rate. In other cases users inadvertently touched another item on the way to the target.

Position: Overall, as in the previous experiment, post-hoc analysis revealed a significant difference between the front (2.6s) and the back items (4s). On the back, the right side (4.3s) was significantly slower than the left side $(3.7 \mathrm{~ms})$. This can be explained as users held the phone with the left hand and most of the selection time corresponds to the visual search (back right items are more difficult to see).

\section{Summary of Exp. 2}

This second experiment allowed us to explore suitable values for the projection volume for direct interaction. Projections smaller than $24 \mathrm{~cm} /$ side improved efficiency. The slight cost in accuracy at smaller volumes suggests that target sizes need to be considered carefully for such displays, and may be limited by the 3D input tracking capabilities. Other forms of feedback for mid-air input, as proposed in [7], could also help improve targeting accuracy. We leave this exploration for future work.

\section{IMPLICATIONS OF EXPERIMENTS 1 AND 2}

From this first exploration of mobile-mounted true-3D displays we identified suitable display parameters along with some display constraints.

\section{Suitable display parameters}

Our two experiments enabled us to identify the suitable values for the explored display factors (angle, distance, volume and position of true-3D content). To improve user search and direct input interaction, a true-3D display should be positioned at an angle between 0 and 45 degrees. The center of the projection should be less than $36 \mathrm{~cm}$ away from the mobile device. The volume of the display should be smaller than a cube of $24 \mathrm{~cm} /$ side. The back area of the display opposite to that of the hand holding the device is difficult to access. This suggests target reaching techniques could be developed for such displays as with large displays or tabletops [30]. We apply these parameters in the design of our applications, as described later.

\section{Implications}

Display Volume: Based on our experiments, mobile true-3D displays may consider a limited volume if users are to rely on direct input and wrist rotation as their primary means of interacting with the display. This limitation suggests that if $3 \mathrm{D}$ content is larger than this volume (for example if displaying a very large map) or if the user wishes to translate 3D objects, the content should be clipped to the boundaries of the volume space. Our applications are restricted to displaying content within such limits. However, alternative methods for space allocation and effective space management need to be considered.

Occlusion: Both viewing and touching the back of the mobile true-3D are particularly difficult. We deduce two main implications from this constraint. First, when creating 3D content for this display, designers should avoid including important content (such as controls or interactive objects) on the back region. The second implication is that to allow easier access to the back region, applications should include rotation techniques for rotating the content of the true-3D and not solely rely on wrist control. The user could then access the back region by rotating the content instead of rotating his wrist.

We use our derived parameters and constraints in the rest of our work: first to frame and define usage scenarios, and second as requirements for our applications.

\section{ILLUSTRATIVE EXAMPLES}

Our implementation of the two representative examples was informed by two participatory design sessions. These sessions helped us define the display configurations for mobile true-3D displays: how the $2 \mathrm{D}$ and the $3 \mathrm{D}$ displays content can be coordinated.

\section{Participatory design sessions}

In both sessions participants filled Post-it notes with their ideas on how users should interact with mobile true-3D. All Post-it notes were read and participants could comment on one another's ideas. We took notes throughout the sessions.

Design session 1: The goal of this first session was to collect general mobile true-3D design ideas. We first asked participants to describe how they envision using mobile true-3D and then to think specifically about the map application and give ideas on display content and interaction. Eight HCI graduate students took part in this session. Participants' ideas for mobile true-3D included: maps, instruction delivery (Chinese calligraphy, sign language), online shopping and 3D sculpting. Concerning the 3D map participants suggested displaying "volumetric buildings" and "3D signposts and landmarks". Several general ideas emerged on the relation between mobile and true-3D displays, such as "2D cross-sections" of the $3 \mathrm{D}$ image, "screen replication" to avoid the fat finger problem and "real pop-ups" in 3D. Concerning the input, some participants would like to touch the true-3D image ("nice to touch 3D") while others imagined ways of indirect interaction ("3D cursor at a distance from the finger").

Design session 2: The goal of the second design session was to collect ideas on how to interact in our 3D environment. Ten students with HCI background participated. On the mobile phone, participants cited "moving/rotating the phone for rotating the true-3D content" and "using the device angle to initiate scale". On 
the true-3D space, some participants indicated the use of 3D gestures such as "pinching" for selecting or translating and using the "distance from the hand in mid-air to the phone" for zooming. A participant also cited using "eye tracking" for rotation, and another one using "face tracking" for translation.

\section{Coordinated display configurations}

From these design sessions and the results from the first exploration, we identified a set of display coordination configurations for mobile true-3D. Our configurations are compliant with the volume constraint identified in the first exploration: we only consider configurations with a limited true-3D volume. We define four dimensions to characterize the coordinated display configurations (CDC) of 3D and 2D displays:

CDC1: Independent (Figure 8-a): both displays show separate content. For example, a GUI on the mobile display used to control the 3D content on the true-3D display.

CDC2: Extruded replication (Figure 8-b): the true-3D display extrudes the content of the mobile display in 3D. For example, the 3D view of a map or spatial workspace.

CDC3: Cross-section (Figure 8-c): the mobile display shows a cross-section view of the true-3D display. An example is views in 3D Computer Aided-Design (CAD) or in architecture visualization.

CDC4: Extruded detail (Figure 8-d): the true-3D display extrudes a detailed $3 \mathrm{D}$ view of a section of the content presented on the mobile display. This corresponds to the idea of a "3D pop-up" in design session 1.

In terms of input, we used three interaction modes: on the mobile, on the true-3D area and mixed true-3D + mobile. Interaction can be single handed (on the mobile) or bimanual (one hand on the mobile, one on the true-3D).

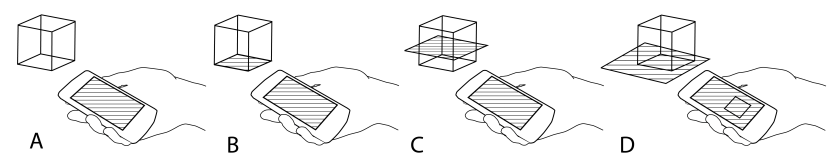

Figure 8. Coordinated Display Configurations of 2D mobile and true-3D displays.

We apply our coordinated display configurations in two applications: the 3D map and the interior design application. Our applications were designed based on the projection parameters we obtained from the first two experiments.
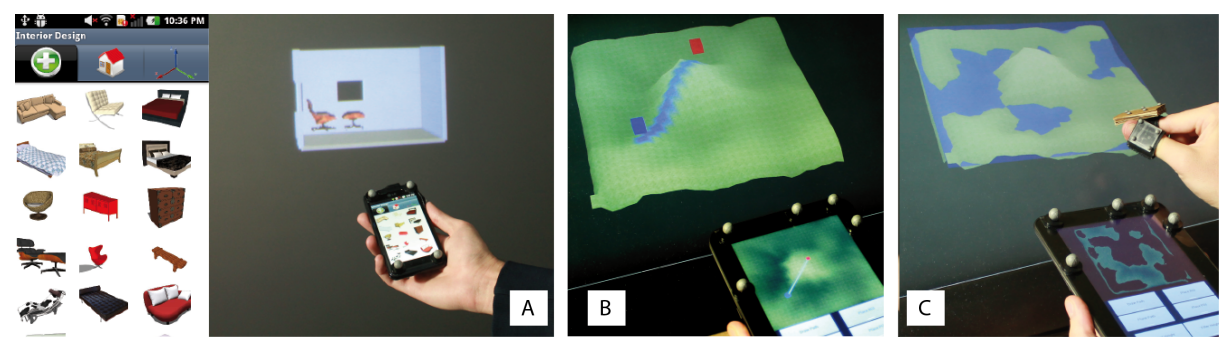

Illustrative application 1: 3D map

3D maps can provide information on terrain height. This is useful for military personnel and hikers, who often traverse areas with many rapid changes in elevation. 3D maps provide advantages over conventional $2 \mathrm{D}$ maps as landmarks on the former closely resemble their physical counterparts. They also decrease mental load in comparison to 2D maps [22]. True-3D display maps enable users to align their viewpoint with objects in the real-world. Such 3D maps are being constructed in projects such as the Urban Photonic Sandtable Display [8].

Our application consists of a top-down view of a 2D map on the mobile device's display, while a 3D version of the map is shown on the true-3D display (CDC2, Figure 9-b). The 3D map is clipped to the volume of the true-3D display (volume constraint from our first exploration). Interactions can be performed through either the touchscreen or by using pinch gestures in the true-3D display volume. Various overlay information, such as paths and points/regions of interest can be placed by interacting with the 3D map. These are represented by visual markers on the 3D map, which are replicated on the 2D map as well. This display relationship allows for modification of markers on one display, with all changes appearing on both displays. This design helps overcome the difficulty of interacting with the back region of the display (occlusion constraint from our first exploration); the $2 \mathrm{D}$ view can be used to verify the position of an out-of-sight marker as it is being placed in 3D. Map content can be filtered based on a number of different properties. In our application, we focus on height filtering. A user can move their hand up or down to control a horizontal selection plane, which intersects the 3D map (CDC3, Figure 9-c). The 2D view displays a horizontal slice of the 3D map at the intersection height. This takes advantage of the 3D and multi-display setup by allowing a visualization of the selected height on the original 3D map, while the $2 \mathrm{D}$ version displays the height-filtered map.

\section{Illustrative application 2: Interior design}

Interior design is a field with a long history in computeraided design (CAD). Recently, with the advent of mobile devices, interior design applications such as AutoCAD provide mobile support with great success (more than 5 million downloads on the Android Market). They facilitate the design process in the field and as a result can integrate features into this process, such as taking pictures of the real

Figure 9. Illustration of the display configurations: A. Independent; B. Extruded replication; C.Cross-section; D. Extruded detail

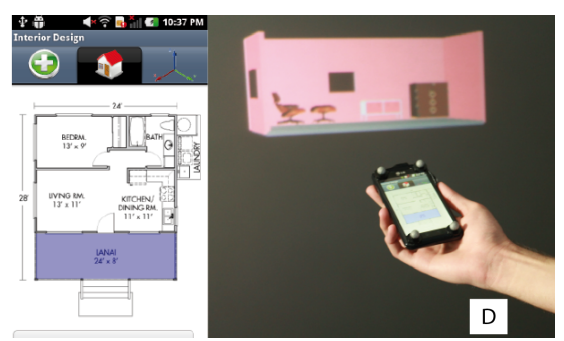


space being designed. An obvious limitation of such applications is the difficulty of manipulating 3D content on a mobile device. Mobile true-3D displays could be used to perform and brainstorm $3 \mathrm{D}$ interior design in the field.

Our interior design application consists of a 3D representation of the room to design and a mobile interface with three views. The room occupies the true-3D volume and cannot be translated outside of it (a solution to accommodate the volume constraint). The front wall is transparent to allow the user to easily inspect and position the $3 \mathrm{D}$ furniture (a solution to the occlusion constraint). The three views of the mobile phone illustrate different aspects of our display configurations: the first view displays a set of images of furniture (CDC1, Figure 9-a). When the user selects furniture, it is added to the $3 \mathrm{D}$ room (it appears in mid-air so that the user can select it and position it). The second view represents the floor plan of the house. On the floor plan, the room currently selected is displayed in a different color (CDC4, Figure 9-d). The user can select another room to change the $3 \mathrm{D}$ room on the true-3D display. The third view allows users to rotate and scale the selected furniture by using a slider or by setting the directtouch mode to "rotate" or "scale" so that the user can rotate or scale the 3D furniture using his/her fingers by pinching. The user can rotate and position the object at the same time by performing a bi-manual interaction: with the right hand he/she directly rotates the object on the 3D environment, while translating the phone can be delegated to the left hand, thus moving the room.

\section{DISCUSSION}

\section{Lessons learned}

Mobile true-3D - opening new usages. Most previous works on MMDE were motivated by the need for expanding mobile displays. One lesson learned from our explorations is that mobile true-3D displays will represent a shift in how users interact with mobile platforms. Accordingly designers will need guidelines to create content for this novel interaction environment.

Design guidelines: Our Coordinated Display Configurations (CDC) can help designers explore design alternatives for usage applications. For example, an application to view 3D photos can be implemented using the Extruded detail configuration (CDC4). A 3D image is shown on the true-3D projection while the mobile phone displays the collection of images. Another view can be based on the Independent configuration (CDC1), for example to provide an interface to also allow editing the image. In reality, various CDCs will co-exist in a single application, as we demonstrated in our two proof-ofconcept applications. By integrating several CDCs in their applications designers will enrich mobile true-3D interfaces and user interaction capabilities.

Display properties: In the context of natural user interactions with mobile true-3D, we can take away the resulting properties that emerged from our first two experiments. These were designed specifically to allow full content viewing through wrist rotation, and interaction using direct input. The use of other interaction techniques with mobile true-3D may allow overcoming some of these constraints: for example, a technique for content rotation can replace natural wrist-based rotation and allow access to occluded elements on the true-3D.

\section{Limitations and future work}

Our work is built upon some assumptions on the capabilities and limitations of future mobile true-3D displays. We assumed mobile true-3D projectors will be able to display a volumetric image of any volume, at any distance and any angle of the phone. We made this assumption to evaluate the user limitations independently from technology capabilities.

The results of our experiments are influenced by the technology we used to emulate the true-3D displays. The obvious differences between this technology and the final true-3D display, in terms of color, brightness or $3 \mathrm{D}$ perception, may alter the results from our experiments. However, most of those results are strongly influenced by human physiological limitations on wrist-based rotation and arm reach. Thus we believe these technical differences do not have a fundamental impact on our findings. Moreover, researchers have used such platforms for developing and testing novel technologies.

There is still much to learn on how mobile true-3D displays will be used in a real mobile situation. A next step in our work will be to use existing mobile augmented reality glasses [28] to evaluate the display properties in mobile situation. A second perspective to our work is to explore the input space of mobile true-3D. In our work we have identified some input configurations and implemented several interaction techniques, including finger gestures such as pinching. We will explore the input configurations space to propose a full suite of interaction techniques. Finally, a third perspective derives from user collaboration on the true-3D display. This will allow us to elaborate on our coordinated display and input configurations.

\section{CONCLUSION}

In this paper we present a two-part exploration of the concept of mobile true-3D. In the first exploration we do the first ever study on mobile true-3D display properties. Results reveal the best distance to phone, angle to phone, display volume and positions on the display for good visual search and direct touch input interaction on mobile true-3D displays. We use these results to guide the design of our applications. Finally, in the last exploration we identify coordinated display configurations between the 2D mobile display and the true-3D display. We define four display configurations named Independent, Extruded replication, Extruded detail and Cross-section. We illustrate those configurations using our two proof-of-concept applications. 
In HCI we find numerous examples of novel technologies whose adoption, from discovery to commercial use, take decades [4]. Buxton refers to this process as the Long Nose of Innovation [3]. Our paper is motivated by our will to reduce the long nose for mobile true-3D. While extensive research is taking place to engineer mobile true-3D $[8,10,16,20]$ we provide a contribution in this vein to identify application guidelines, limitations and scenarios to help future adoption of this technology.

\section{REFERENCES}

1. Bergé, L.-P., Serrano, M., Perelman, G., and Dubois, E. 2014. Exploring Smartphone-Based Interaction with Overview+Detail Interfaces on 3D Public Displays. In Proc. of MobileHCI '14, ACM, 10 pages.

2. Butler, A. et al. 2011. Vermeer: direct interaction with a $360^{\circ}$ viewable 3D display. In Proc. of UIST '11, ACM, 569-576.

3. Buxton, B. The Long Nose of Innovation. CHI 2011 Keynote.

4. Buxton, B. Buxton Collection (last accessed February 2014). http://research.microsoft.com/buxtoncollection

5. Cauchard, J., et al. 2011. Visual separation in mobile multi-display environments. In Proc. of UIST'11, ACM, 451-460.

6. Cauchard, J., et al. 2012. Steerable projection: exploring alignment in interactive mobile displays. Personal Ubiquitous Comput. 16, 1 (January 2012), 27-37.

7. Chan, L. et al. 2010.Touching the void:direct-touch interaction for intangible displays. In Proc. of CHI'10, ACM, 2625-2634.

8. DARPA (last accessed February 2014), http://www.darpa.mil/newsevents/releases/2011/11/23.a $\underline{\operatorname{spx}}$

9. Ens, B., Finnegan, R. and Irani, P. 2014. The Personal Cockpit: A Spatial Interface for Effective Task Switching on Head-Worn Displays. In Proc. of CHI '14. ACM, 10 pages (to appear).

10. Fattal, D. et al. 2013. A Multi-Directional Backlight for a Wide-Angle Glasses-Free Three-Dimensional Display. In Nature 495, 348-351 (21 March 2013).

11. Froehlich, B. et al. 2006. The GlobeFish and the GlobeMouse: two new six degree of freedom input devices for graphics applications. In Proc. of CHI '06, ACM, 191-199.

12. Hancock, M. et al. 2007. Shallow-depth 3d interaction: design and evaluation of one-, two- and three-touch techniques. In Proc. of CHI'07, ACM, 1147-1156.
13. Hancock, M., Ten Cate, T., and Carpendale, S. 2009. Sticky tools: full 6DOF force-based interaction for multi-touch tables. In Proc. of ITS '09, ACM, 133-140.

14. HelioDisplay, http://www.io2technology.com

15. Hilliges, O., Kim, D., Izadi, S., Weiss, M. and Wilson, A. 2012. HoloDesk: direct 3d interactions with a situated see-through display. In Proc. of CHI '12, ACM, 2421-2430.

16. Karlsson, $\mathrm{N}$ et al. 2012. iAR: an exploratory augmented reality system for mobile devices. In Proc. of VRST '12, ACM, 33-40.

17. Kimura, H., et al. 2011. True 3D display. In $A C M$ SIGGRAPH 2011 Emerging Technologies. ACM, Article 20, 1 pages.

18. Kratz, S. et al. 2012. PalmSpace: continuous arounddevice gestures vs. multitouch for 3D rotation tasks on mobile devices. In Proc. of AVI'12, ACM, 181-188.

19. Martinet, A., Casiez, G. and Grisoni, L. 2010. The effect of DOF separation in 3D manipulation tasks with multitouch displays. In Proc of VRST '10. ACM, 111-118.

20. Nii, H. et al. 2012. Fuwa-Vision: an auto-stereoscopic floating-image display. In SIGGRAPH Asia 2012 Emerging Technologies. ACM, Article 13, 4 pages.

21. Nintendo 3ds, http://www.nintendo.com/3ds

22. Nurminen, A. and Oulasvirta, A. 2008. Designing interactions for navigation in 3D mobile maps. In Lecture Notes in Geoinformation and Cartography, Springer, pp. 198-224

23. Rahman, M., Gustafson, S., Irani, P. and Subramanian, S. 2009. Tilt techniques: investigating the dexterity of wrist-based input. In Proc. of CHI'09, ACM, 1943-52.

24. Rashid, U. Nacenta, M. and Quigley, A. 2012. The cost of display switching: a comparison of mobile, large display and hybrid UI configurations. In Proc. of AVI '12, ACM, 99-106.

25. Rukzio, E., Holleis, P. and Gellersen, H. 2012. Personal Projectors for Pervasive Computing. IEEE Pervasive Computing 11, 2 (April 2012), 30-37.

26. Samsung Flexible Amoled vision (last accessed Feb. 2014), http://youtube.com/watch?v=f4AhTiQkWwk

27.Visbox, http://www.visbox.com/

28.Vuzix, http://www.vuzix.com/home/

29. Song, P. et al. 2011. WYSIWYF: exploring and annotating volume data with a tangible handheld device. In Proc. of CHI'11, ACM, 1333-1342.

30. Winkler, C., Pfeuffer, K. and Rukzio, E. 2012. Investigating mid-air pointing interaction for projector phones. In Proc. of ITS '12, ACM, 85-94. 